\title{
The colliding epidemics of COVID-19, Ebola, and measles in the Democratic Republic of the Congo
}

The Democratic Republic of the Congo is facing major public health challenges due to a confluence of major outbreaks of Ebola virus disease, measles, and COVID-19. ${ }^{1-4}$ The tenth Ebola outbreak in eastern DR Congo began on Aug 1, 2018, and as of May 28, 2020, there have been 3406 Ebola virus disease cases with 2243 deaths. The Ebola virus disease outbreak was well controlled in northeast DR Congo following a multisectoral response, but four new confirmed Ebola cases were detected in northwest DR Congo on June 1,2020 , and an outbreak response is underway. ${ }^{4}$ Additionally, the DR Congo has been burdened with recurrent measles outbreaks: 133802 cases in 2011, 88381 cases in 2013, and 311471 cases in 2019. ${ }^{2}$ The first confirmed case of COVID-19 in DR Congo was diagnosed on March 10, 2020, and the government declared a state of emergency on March 24, 2020. A national multisectoral response committee instituted lockdown in the capital, Kinshasa, the epicentre of the epidemic in DR Congo, in which daily confirmed cases now average 100. As of June 16, 2020, 4777 COVID-19 cases with 106 deaths have been reported from the DR Congo. ${ }^{5}$

Although the COVID-19 pandemic presents unique challenges that threaten the health, economy, and social fabric of DR Congo, several lessons learned from dealing with the devastating Ebola virus disease outbreaks have been invaluable and are guiding the ongoing COVID-19 public health response. ${ }^{6}$ These responses include involvement of community leaders and institutions with communication to the public in local languages to explain the disease and its prevention strategies and disseminating messages in terms that are culturally understandable. Ebola virus disease control infrastructure, protocols, and staff have been repurposed and applied to the COVID-19 response. Multidisciplinary teams of nurses, doctors, medical students, and community health-care workers are implementing COVID-19 sensitisation, screening, and testing activities endorsed by the Ministry of Health and community and religious leaders?.

All COVID-19 testing by RT-PCR is done at the National Institute of Biomedical Research in Kinshasa. Efforts to decentralise testing to provinces and improve turnaround are underway using point-of-care testing with the GeneXpert platform, but this remains challenging because of cost and supply constraints for severe acute respiratory syndrome coronavirus 2 testing cartridges. Several hospitals were identified as reference centres for the treatment of COVID-19, and intensive care units have been resourced with additional ventilators and oxygen supplies as part of the national plan. A clinical protocol has been developed by the Case Management Commission with support from technical partners. Bilateral and multilateral partnerships provided donations of medical equipment to hospitals, and clinical staff are being trained on COVID-19 case management. Patients with moderate and severe COVID-19 are hospitalised for supportive care, oxygen therapy, and anticoagulation as per WHO guidelines. ${ }^{8}$

The strong national commitment in DR Congo to the COVID-19 and Ebola virus disease epidemics provides hope that alignment of public health responses will prevent the high morbidity and mortality seen in South Africa. The DR Congo government understands the importance of strengthening its health systems through establishing effective partnerships with international stakeholders who leverage their unique areas of expertise to achieve tangible results. However, as with other African countries, major challenges for COVID-19 control remain. Mitigation measures such as travel bans and lockdowns in DR Congo are having negative socioeconomic effects on the population. Indeed, most citizens are unemployed and live below the poverty line in shanty towns or rural settings in overcrowded housing, which makes COVID-19 preventive measures, such as hand washing and social distancing, difficult. Also, the high prevalence of comorbidities, including chronic conditions such hypertension, diabetes, HIV and AIDS, and tuberculosis, add to the COVID-19 mortality risk. ${ }^{9}$ Although attention has been focused on COVID-19 and Ebola virus disease, there have also been 369520 measles cases with 6779 deaths in the past year, which illustrates that vaccination efforts have been suboptimal because of disruptions to immunisation campaigns and suggests that there are potential threats of resurgence of other vaccine-preventable infectious diseases.
Published Online June 23, 2020 https://doi.org/10.1016/ S2214-109X(20)30281-3 
DR Congo's early COVID-19 mitigation interventions have allowed the multisectoral response committee to gain time and prepare for the anticipated COVID-19 peak, but its response is being implemented in a fragile health system that faces challenges, including the coexisting Ebola virus disease and measles outbreaks. The World Bank provided US\$300 million to support the 2018-2020 Ebola virus disease response strategy, and it should ensure that additional assistance includes support for primary health care and regular supply of essential medicines, vaccines, and technical assistance. ${ }^{10}$ Although urgent measures are required to slow the spread of COVID-19 and tackle the renewed threat of Ebola virus disease, every effort must be made by stakeholders to ensure that prevention programmes for measles or other infectious diseases, including transporting vaccinations for children, are not compromised.

JBN reports grants from US National Institutes of Health and the Bill \& Melinda Gates Foundation. AZ reports funding from the European Developing Countries Clinical Trials Partnership and is in receipt of a NIHR Senior Fellowship Award. J-JMT-F is leading the COVID-19 Multisectoral Response Committee in Democratic Republic of the Congo (DR Congo). All other authors declare no competing interests. JBN, PM-K, and JO contributed equally as first authors. $A Z$ and J-JMT-F contributed equally as joint senior authors. The views and opinions expressed in this article are those of the authors and do not necessarily reflect the official view or position of their government, organisation, employer, or institution. We acknowledge critical review by John Johnson, Case Western Reserve University, Cleveland, $\mathrm{OH}, \mathrm{USA}$.

Copyright $\odot 2020$ The Author(s). Published by Elsevier Ltd. This is an Open Access article under the CC BY 4.0 license.

* Jean B Nachega, Placide Mbala-Kingebeni, John Otshudiema, Alimuddin Zumla, Jean-Jacques Muyembe Tam-Fum jbn16@pitt.edu

Department of Medicine and Centre for Infectious Diseases, Stellenbosch University Faculty of Medicine and Health Sciences, Cape Town, South Africa
(JBN); Department of Epidemiology and International Health, Johns Hopkins Bloomberg School of Public Health, Baltimore, MD, USA (JBN); Department of Epidemiology, Infectious Diseases and Microbiology, and Center for Global Health, University of Pittsburgh, Pittsburgh, PA 15261, USA (JBN); National Institute of Biomedical Research and Department of Medical Microbiology and Virology, Faculty of Medicine, University of Kinshasa, Kinshasa, Democratic Republic of Congo (PM-K, J-JMT-F); World Health Organization, Health Emergencies Program, COVID-19 Response, Epidemiological Surveillance Team, Democratic Republic of Congo (JO); Department of Infection, Division of Infection and Immunity, Centre for Clinical Microbiology, University College London, London, UK (AZ); and National Institute for Health Research Biomedical Research Centre, University College London Hospitals, London, UK (AZ)

1 Nsio J, Kapetshi J, Makiala S, et al. 2017 outbreak of ebola virus disease in northern Democratic Republic of Congo. J Infect Dis 2020; 221: 701-06.

2 llunga Kalenga O, Moeti M, Sparrow A, Nguyen VK, Lucey D, Ghebreyesus TA. The ongoing Ebola epidemic in the Democratic Republic of Congo,2018-2019. N EnglJ Med 2019; 381: 373-83.

3 WHO. Measles and Rubella Surveillance Data. 2020. https://www.who.int/ immunization/monitoring_surveillance/burden/vpd/surveillance_type/ active/measles_monthlydata/en/ (accessed June 7, 2020).

4 The Washington Post. New Ebola outbreak declared in Congo city that last saw the virus in 2018. 2020. https://www.washingtonpost.com/world/ africa/new-ebola-outbreak-declared-in-congo-city-that-last-saw-thevirus-in-2018/2020/06/01/33a9f958-a3ff-11ea-898e-b21b9a83f792 story.html (accessed June 7, 2020)

5 WHO. Coronavirus disease (COVID-2019) situation reports. 2020. https://www.who.int/docs/default-source/coronaviruse/situationreports/20200615-covid-19-sitrep-147.pdf?sfvrsn=2497a605_4 (accessed June 16, 2020)

6 Mobula LM, Samaha H, Yao M et al. Recommendations for the COVID-19 response at the national level based on lessons learned from the Ebola virus disease outbreak in the Democratic Republic of the Congo. Am J Trop Med Hyg 2020; published online May 19. https://doi.org/10.4269/ajtmh.20-0256.

7 Nachega JB, Grimwood A, Mahomed H, et al. From easing lockdowns to scaling-up community-based COVID-19 screening, testing, and contact tracing in Africa - shared approaches, innovations, and challenges to minimize morbidity and mortality. Clin Infect Dis 2020; published online May 31. https://doi.org/10.1093/cid/ciaa695.

8 WHO. Clinical Management of COVID-19. 2020. https://www.who.int/ publications-detail/clinical-management-of-covid-19 (accessed May 31, 2020).

9 Mehtar S, Preiser W, Lakhe NA, et al. Limiting the spread of COVID-19 in Africa: one size mitigation strategies do not fit all countries. Lancet Glob Health 2020; published online April 28. https://doi. org/10.1016/S2214-109X(20)30212-6

10 Wadman M. World Bank dedicates $\$ 300$ million to Ebola response. 2019 https://www.sciencemag.org/news/2019/07/world-bank-dedicates-300million-ebola-response (accessed June 7, 2020). 